\title{
Plasmon-Assisted Energy Transfer in Hybrid Nanosystems
}

\author{
Mareen Glaeske ${ }^{1}$, Sabrina Juergensen ${ }^{1}$, Luca Gabrielli², Enzo Menna ${ }^{2}$, Fabrizio Mancin², \\ Teresa Gatti ${ }^{2}$, and Antonio Setaro*1 \\ ${ }^{1}$ Department of Physics, Free University Berlin, Arnimallee 14, 14195 Berlin, Germany \\ ${ }^{2}$ Department of Chemical Sciences, University of Padova, via Marzolo 1, 35131 Padova, Italy
}

\begin{abstract}
While direct optical excitation of carbon nanotubes activates only the tube species strictly matching the excitation source, excitation energy transfer processes provide a single excitation channel for all the nanotubes species in a sample. The requirement of an overlap between donor emission and acceptor absorption limits the poll of donors able to trasfer their excitation to the tubes, leaving the high-energy part of the solar spectrum excluded from such processes. Here we show that the grafting of small metal nanoparticles to the tubes alters those rules, enabling energy transfer process from molecules for which the standard energy transfer process is strongly suppressed. We demonstrate the onset of an energy transfer band in the UV/blue spectral region for an hybrid gold-pyrene-nanotube system, yielding collective emission from all the tubes present in our samples upon excitation of pyrene.
\end{abstract}

\section{Introduction}

With tiny diameters of few nanometres and lengths up to centimetres, single-walled carbon nanotubes (SWNTs) bridge quantum mechanics to the macroscopic world. They establish ballistic conduction channels [1] and their room-temperature optical response is ruled by strongly-bound coherent excitons $[2,3]$. Favoured by an outstanding mechanical strength and durability $[4,5]$, SWNTs uphold benefits for a huge plethora of advanced purposes: For example, they establish single-photon sources at room temperature [6] and are embedded into photo-detectors and photovoltaic devices [7, 8], as well as in near-infrared organic light emitting diodes [9].

As-produced SWNT batches contain several species ${ }^{1}$ and the isolation process to yield big amounts of a single species is still challenging. The principle constrain of SWNTs is the sharp selectivity of their direct-optical excitation: Given a certain tube species, only photons with a specific wavelength are able to optically excite this unique species [10]. Together with the distinctive wavelength of the emitted photons, this excitation wavelength characterizes one specific nanotube species associated with $\vec{c}$ [5]. The direct optical excitation process of SWNT sets is therefore quite inefficient, since only a small fraction of the total nanotube population in a commercially available nanotube batch (of different species) gets activated by one certain excitation wavelength. There is anyway an alternative process to circumvent such limitation. It involves an indirect excitation path leading to the same result as the direct optical excitation, i.e. recombinative emission from SWNTs. Such indirect optical excitation process is mediated by molecules in close proximity to SWNTs. It bypasses the limitations of the direct one, providing an excitation pathway for all the nanotubes species present in a sample [11].

\footnotetext{
${ }^{*}$ Corr. author: setaro@physik.fu-berlin.de

${ }^{1} \mathrm{~A}$ nanotube species is uniquely identified by the so-called chiral vector $\vec{c}=\left(n_{1}, n_{2}\right)$, which specifies the length and direction of the nanotube folding expressed in the graphene unit vectors base.
} 
(a)

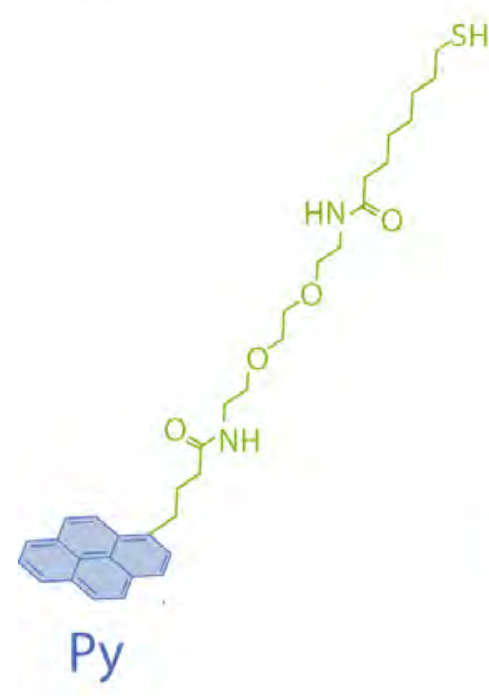

(b)

(c)

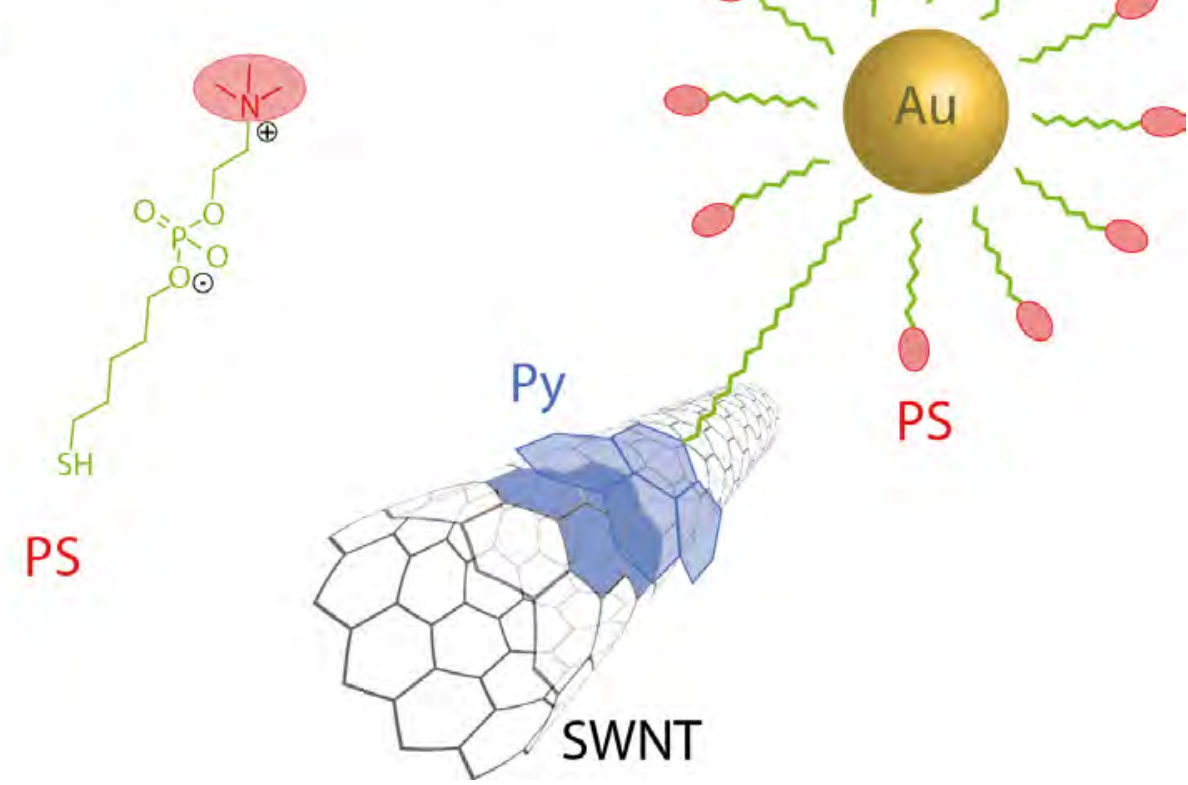

Figure 1: (a) Molecular sketch of the pyrene-derivative Py. The pyrene head is higlighted in blue. (b) Molecular sketch of phosphorylcholine PS, used to stabilize the AuNPs in suspension. The watersoluble head is highlighted in red. (c) Sketch of the AuPy@NT hybrids comprising SWNTs decorated with AuPy particles.

Once granted the proper coating conditions, the indirect optical process establishes an additional single-excitation-wavelength channel. The process encompasses the optical excitation of the molecule, the transfer of its excitation to the tubes, and the radiative emission from all the molecule-coated nanotubes $[11,12]$. The efficiency of such process approaches $100 \%[13,14]$. Successful molecule-mediated excitation transfer with collective emission from all the SWNT species has been successfully observed so far for perylene $[11,14,15,16]$ and porphyrin $[12,13,17,18,19,20]$, small aromatic fragments whose emission regions span through the green-red part of the visible spectrum and overlap with the excitation window of the most common commercially available SWNTs [21]. For light-harvesting purposes, however, it would be useful to extend the spectral range of the excitation transfer to cover the region around the maximum of the solar spectrum. This could have favorable impact on technologies relying on down-conversion of light. A valid candidate is pyrene, a small aromatic molecule composed by four benzene rings, highlighted in blue in the molecular sketch of Figure 1. Pyrene, with an excitation window in the UV region and emission in the blue, displays the right optical features for such purposes. Its flat morphology, moreover, makes it strongly stick onto the SWNTs sidewalls through $\pi-\pi$ stacking interactions without the aid of any additional items [22, 23, 24, 25]. Tethered pyrene has been exploited as glueing element to attach functionalities for photoconversion purposes to single- and multi-walled carbon nanotubes [26, 27, 28, 29]. Pyrene, contrarily to perylene and porphyrin, has not been shown to induce radiative emission from the tubes through excitation transfer yet. This is mainly due to the fact that the underlying excitation energy transfer process involves an energetic match to transfer the excitation between a donor (the molecule) and an acceptor (the nanotube) [30]: Such process is efficent only if the emission spectrum of the donor and the excitation spectrum of the acceptor overlap significantly, which is not the case of pyrene and SWNTs. Recently, it has been demonstrated that plasmon-assisted energy transfer overcomes the usual constraints of the excitation transfer process [31]. The presence of the plasmon affects the electromagnetic environment surrounding the donor and acceptor and significantly alters the nature of their coupling [32]. In the following we will show our results on metallic nanoparticles-activated energy transfer from pyrene to SWNTs. 

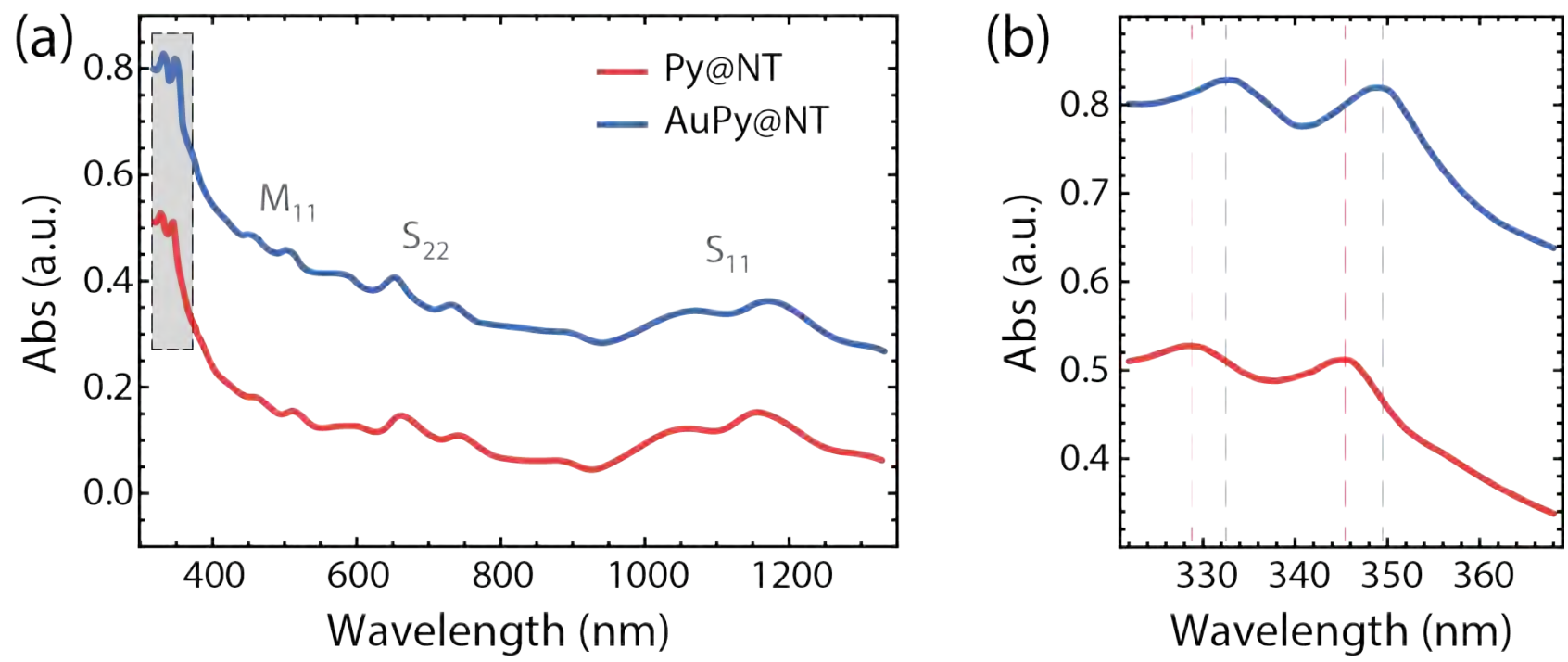

Figure 2: (a) Comparison between the absorption spectra of Py@NT (red curve) and AuPy@NT (blue curve). (b) Enlarged view of the comparison between the pyrene absorption bands of the two samples in the region highlighted by the gray box in panel (a). An offset of 0.15 has been added to the AuPy@NT curve.

\section{Experimental details and sample preparation}

We prepared our samples following the routine established in our previous study [33], yielding gold nanoparticles (AuPy) with diameters of $2 \mathrm{~nm}$, coated with a mixture of the pyrene derivative Py (sketched in Figure 1a) and and a phosphorylcholine derivative PS (sketched in Figure 1b). The latter, with his water soluble head, stabilize the AuNPs in water. The monolayer covering AuPy is composed by $10 \%$ Py and $90 \%$ PS. For further details on the synthesis and characterization of the particles as well as their grafting onto the tubes, please refer to our past publications, where we focused on the proof of the effective hybridization of the tubes with the AuNPs [34] and on the optimization of the sample preparation to achieve high emission yields [33].

Consistently with our previous study, we used CoMoCAT single-walled nanotubes by SouthWest NanoTechnologies (SWeNT), batch SG76, with diameter of (0.9-1.2) nm and high aspect ratio $(\geq 1000)$. Following the same water-suspension procedure of [33], based on a variation of the standard mild sonication/centrifugation procedure $[35,36]$, we decorated our SWNTs, respectively, with the pyrenederivative Py to yield the hybrid Py@NT and with the pyrene-decorated gold nanoparticles AuPy to yield the hybrid AuPy@NT. To ensure comparability between the intensities and the emission yields, the suspensions were prepared to grant the same amount of nanotubes and pyrene in all the samples, please refer to Ref. [33] for the indications on starting concentrations.

2- $d$ excitation-emission spectroscopy was performed through an Horiba Fluorolog system equipped with a Xe-arc lamp and a CCD detector for the measurements in the UV/Vis range and a liquid nitrogen-cooled InGaAs detector for measurements in the IR range. The absorption spectra were taken with a Perkin-Elmer Lambda 950 spectrophotometer.

Figure 2 reports the absorbance of the nanotubes coated with, repectively, pyrene (Py@NT, red curve) and pyrene-gold complexes (AuPy@NT, blue curve). An offset of 0.15 has been added to the AuPy@NT curve. The spectra exhibit the characteristic absorption bands for metallic (higlighted as $M_{11}$ for sake of clarity) and semiconducting (higlighted as $S_{11}$ and $S_{22}$ ) SWNTs. The gray area in Figure 2a highlights the pyrene absorption region and is zoomed in Figure 2b for better visibility. The different bands in both spectra display similar amplitudes, confirming a comparable amount of tubes and pyrene in both samples. Noteworthily, the distinctive plasmonic absorption band of Au at the frequency corresponding to the threshold of the interband transition (ca. $530 \mathrm{~nm}$ ) is not to be 
observed for particles with such small diameters, where quantum confinement changes the behaviour of the free electrons. Since the quantization energy exceeds the plasmon frequency, such small metallic particles do not show the distinctive Au plasmon absorption band in UV/Vis spectroscopy [37].

\section{Discussion and results}

Several strategies have been pursued to control and mold the flow of energy at the nanometric level in multipartite systems. To enhance the emission from an ensamble of identical emitters, for example, Dicke proposed to exploit superradiance arising from coherent states established by the exchange of virtual photons [38]. Superradiance from Dicke-like phenomena has been obtained in several nanosystems [39] but, for the cooperativity to take place, it is required to have an ensamble of identical emitters exchanging virtual photons at a unique and very specific wavelength. Plasmon-assisted phenomena, on the contrary, have proven to grant more flexibility than purely photonic effects. The coupling among emitters in the plasmonic Dicke effect occurs through the exchange of virtual plasmons [40]. The surface-extended nature of plasmons in metallic nanoparticles ensures that all the emitters are coupled with equal strength, making the interparticle cross-talk more uniform and robust than for the photonic case [40,41]. Plasmons are broad-banded and ensure the interaction of emitters with different sizes and resonances [42] and extend the range of excitation transfer to bigger separations than the typical Förster ranges [43]. The Förster mechanism is a two particle process where an optically excited donor transfers its excitation to an acceptor. The so-called Förster radius $R_{0}$ is the characteristic parameter at which half of the luminescence of the donor is quenched by the acceptor [44], defined as:

$$
R_{0}=0.2108\left[\kappa^{2} n^{4} \Phi_{0} \int I_{d}(\lambda) \epsilon_{a}(\lambda) \lambda^{4} d \lambda\right]^{\frac{1}{6}}
$$

where $n$ is the refractive index of the embedding medium, $\Phi_{0}$ the intrinsic fluorescence quantum yield of the donor, $\kappa$ an orientational factor between the normalized donor $\left(\hat{\mu}_{d}\right)$ and acceptor $\left(\hat{\mu}_{a}\right)$ transition dipoles, defined as

$$
\kappa=\hat{\mu}_{d} \cdot \hat{\mu}_{a}-3\left(\hat{\mu}_{d} \cdot \hat{r}_{d a}\right)\left(\hat{\mu}_{a} \cdot \hat{r}_{d a}\right),
$$

$I_{d}(\lambda)$ is the normalized emission spectrum of the donor, and $\epsilon_{a}(\lambda)$ the molar extinction coefficient of the acceptor. It is worth pointing out that, in the standard picture of the Förster process, the transfer takes place only under a configurational (the dipole of the donor and the acceptor are not mutually orthogonal) and energetic match (there is a significant overlap between the emission of the donor and absorption of the acceptor) [44]. While the configurational match can be optimized by controlling the particles' morphology [15], little can be done in binary systems embedded in homogeneous media and zero overlap integral like in our case, where the emission of the donor (pyrene, 360-430 nm [25]) does not overlap with the absorption of the acceptors (SWNTs, 570-900 nm, depending upon the tube species [21]). Please note that, in case of close proximity between acceptor and donor, the theoretical framework to depict such processes becomes much more complicate than the Förster one. An energetic alignement between the donor and acceptor bands, though, is still a key parameter for the excitation transfer to occurr.

The presence of complex dielectric systems, such as plasmonic particles, changes the local nature of the electromagnetic fields. Recently, Hsu et al. demonstrated that in non-homogeneous, dispersive, or absorbing environments the donor-acceptor coupling can override the rules of the excitation transfer mechanism [32]. They showed that the transition amplitude for the energy transfer can be written as

$$
M\left(\vec{r}_{d}, \vec{r}_{a}, \lambda\right)=-\vec{\mu}_{a} \cdot \vec{E}_{d}\left(\vec{r}_{a}, \lambda\right),
$$

where $\vec{E}_{d}\left(\vec{r}_{a}, \lambda\right)$ is the total electric field (comprising the plasmon contribution) generated by the donor in the position of the acceptor ${ }^{2}$. The generalized excitation transfer is ruled by the local behaviour of total electric field, which is determined not only by the donor but also strongly shaped up by the

\footnotetext{
${ }^{2}$ In the case of ideal hertzian dipoles embedded in media with purely real dielectric constants, those results simplify to the standard Förster case [32].
} 
plasmonic system. Hence, the maximum enhancement does not necessarily occur in the donor-acceptor overlap window but rather where the coupling factor (comprising the plasmon contribution) is more efficient, typically in the spectral region of the active plasmonic mode. The strong local enhancement of the plasmonic near-fields in the proximity of the metallic particles drives the transfer process and activates novel excitation channels. Evolved sources like quadropolar plasmonic modes and other dark processes, not detectable with standard optical far-field techniques [45], rule the phenomenology of the excitation transfer. In the case analyzed by Hsu et al. the highest enhancement occurs close to the quadrupolar oscillation mode of the metallic nanoparticle. Here arises a clear difference between nearfield and far-field excitation processes: Quadrupolar oscillations are not dominant in the absorption far-field spectra but nevertheless rule the phenomenology of the near-field mediated excitation transfer.

Our AuNPs have an average diameter of $2 \mathrm{~nm}$ and lie in the intermediate region between single atoms and bulk systems. Their plasmonic features are not detectable with standard far-field UV/Vis spectroscopy (cfr. Figure 2); quantum size effects increase the energy of electron oscillation of small particles with respect to the ones of the bulk case, as well as activate UV inter- and intraband transitions which are normally not detectable $[37,46]$. Those effects push the plasmonic activity of the AuNPs towards the blue/UV spectral region, in which pyrene is optically active. Even if not directly measurable by standard absorption spectroscopy, the plasmonic modes of small metallic clusters are nevertheless active. They for example allow catalytical process to tale place [47] or contribute to the enhancement of the SWNTs' emission [33]. To check whether the presence of AuNPs allows the transfer of excitation from pyrene to the SWNTs, we prepared and compared a set of different samples: Pristine nanotubes without any pyrene, treated with the standard commercial surfactant sodium cholate (we refer to them as the sample SWNTs in the rest of this manuscript), nanotubes coated with pyrene without any Au (sample Py@NT), and nanotubes coated with pyrene and gold (sample AuPy@NT). Figure 1 displays a sketch of the different systems used in our experiment. While the absorption spectra of the samples look very similar to each other (cfr. Figure 2), the emissive response of each hybrid displays its distinctive different response. To this purpose, we performed 2- $d$ excitation-emission (PLE) spectroscopy of our samples covering a broad excitation range, spanning from the UV up to near infrared. In the PLE chart of the pristine SWNTs (Figure 3a) the emission spots of the SWNTs that appear due to the direct-optical $S_{22}$ excitation are recognizable in the visible excitation range. In the UV excitation range, moreover, we can observe the spots due to higher-order $\left(S_{33}\right)$ direct-optical transitions [5]. A similar behaviour is exhibited by the pyrene-coated nanotubes (Py@NT, Figure 3b), where only the emission spots due to the direct-optical excitation can be observed. The lack of spectral overlap between pyrene emission and tubes' absorption hinders any excitation transfer process in this sample, consistently with previous observations in literature on pyrene-based systems [24, 25, 48, 49]. Adding gold to the binary pyrene-nanotube system markedly changes the physical response of our hybrids. The presence of the plasmonic particles enables the transfer of excitation from pyrene to the SWNTs, as revealed by the strong energy-transfer band arising in the UV excitation window of Figure 3c. Please note that this process is activated only when all the three components (pyrene, SWNTs, and AuNPs) interact among each other. To highlight this, we compared in Figure 3d the emission lines of the samples excited at $350 \mathrm{~nm}$. Those lines correspond to the single horizontal lines highlighted in Figure 3, panels a-c. In this excitation range, the emission due to higher-order direct-optical excitation can be detected in the pristine SWNT sample (black line). Likewise, the pyrene-coated sample Py@NT (red line) only displays emission from higher-order directoptical excitation process. The addition of AuNPs to the system activates the energy transfer process. Strong emission through the pyrene-mediated indirect excitation can be observed in the AuNP@NT sample. Please note that the energy-transfer bandsare much broader than for binary molecule-tubes systems $[11,13]$, probably due to the stronger scattering from the AuNPs. Superimposed with the energy transfer signal, a small modulation due to the emission bands from the direct optical excitation of the nanotubes can also be recognized in this sample, suggesting the efficiency of the energy transfer process to be higher than the direct excitation for higher-order transitions. A direct quantification of the absolute yields of these processes is anyway challenging and out of the scope of this manuscript, as it would require very unusual combination of sources, optics, and detectors to cover a wide spectral range from the UV up to the infrared. As a last check, no emission can be observed from the pure 

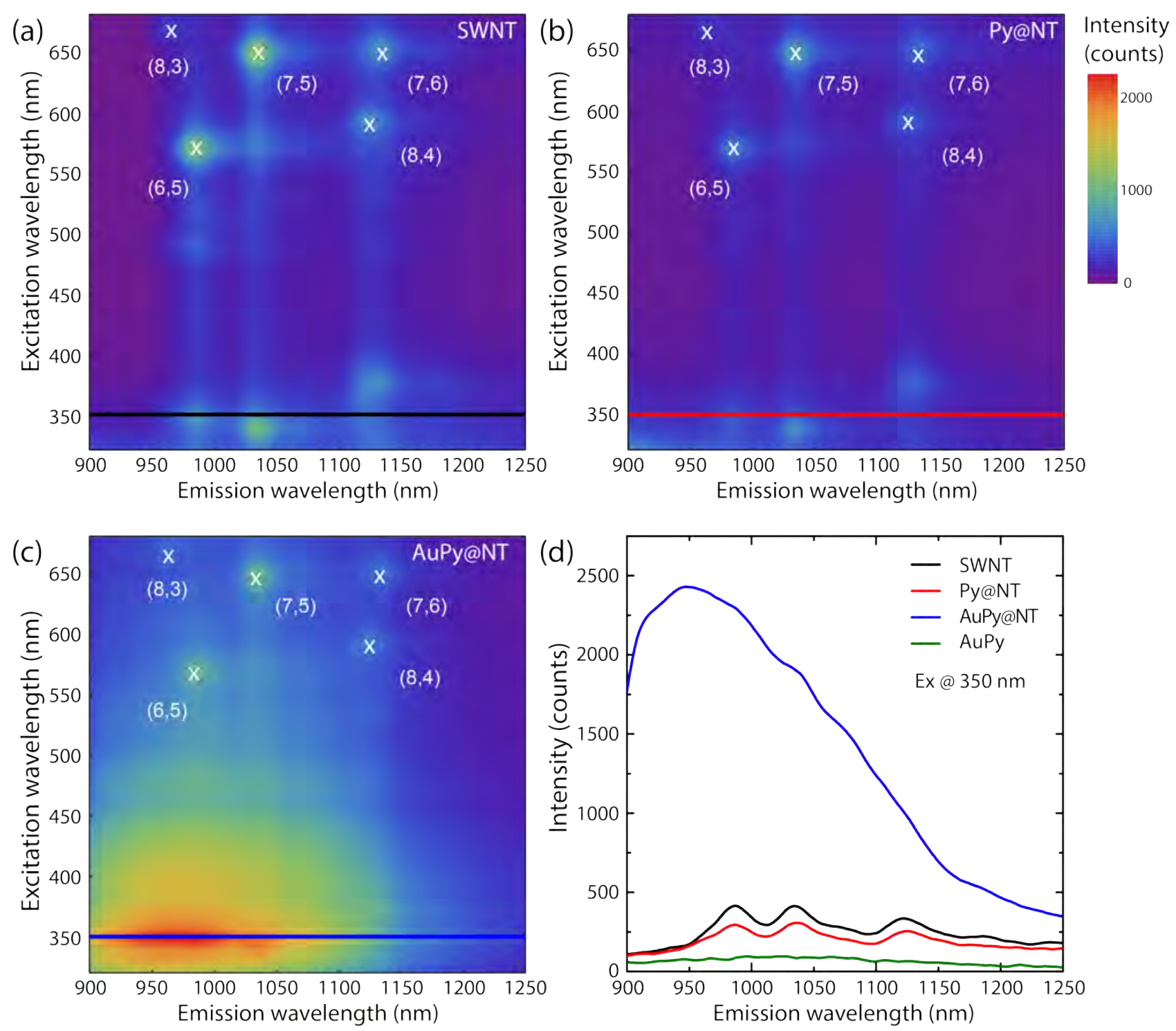

Figure 3: Comparison between the 2- $d$ excitation-emission charts of (a) SWNT, (b) Py@NT, and (c) AuPy@NT samples. (d) Comparison between the emission line of the SWNT (black line), Py@NT (red line), AuPy@NT (blue line), and AuPy (green line) samples excited at $350 \mathrm{~nm}$.

AuPy sample without any SWNTs (green line in Figure 3d), ruling out contributions from other spurious signals to the observed energy transfer emission.

Additional information about our system can be gained by comparing the optical response of pyrene instead of the one from the SWNTs. In Figure 4a, the PLE chart of AuPy carried out in the pyrene emission region (blue-green) reveals several emission bands. Pyrene's photoluminescenece, similarly to other polyaromatic compounds, occurs by its excitation from the ground state to one of the vibrational sub-levels of the first electronic state [50]. The characteristic vibronic sub-bands structure of pyrene monomers occur in the emission region spanning from $360 \mathrm{~nm}$ to $430 \mathrm{~nm}$ of Figure $4 \mathrm{a}$. In the same chart, an additional band due to excimers can be recognized in the $440 \mathrm{~nm}$ to $560 \mathrm{~nm}$ emission range, consistent with previous observations in literature [51]. The direct emission from the pyrene monomer and excimer can be confronted with the indirect emission mediated through the nanotubes (emission region $900 \mathrm{~nm}$ to $1200 \mathrm{~nm}$, Figure 4b). A single excitation line (i.e. a vertical section) in a PLE chart carries information about the ability of the system of being optically excited and hence about its absorbance [52]. Unlike UV/Vis spectrophotometry, excitation spectroscopy allows to monitor a specific process by selecting its characteristic emission line. This is very useful in systems like our, with different optical processes occurring in the same excitation range but displaying different emission wavelengths. Standard absorption spectrophotometry would not be able to disentangle them. 

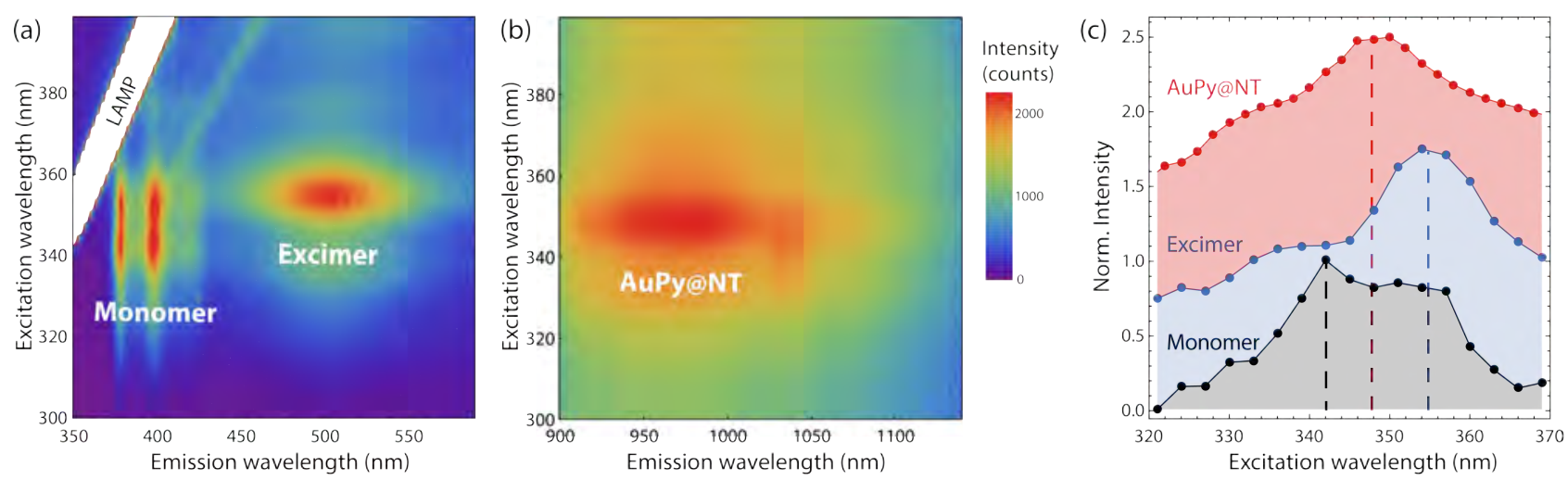

Figure 4: (a) PLE chart of AuPy nanoparticles, displaying emission from pyrene in its monomeric and excimeric form. (b) PLE chart of the AuPy@NT hybrids, displaying the energy transfer band from pyrene to the SWNTs. (c) Single excitation spectra of the monomer (black line, emission at $400 \mathrm{~nm}$ ), excimer (blue line, emission at $500 \mathrm{~nm}$ ), and of the energy transfer band (red line, emission at $980 \mathrm{~nm})$.

Figure 4c compares the single excitation lines of the monomer, excimer, and SWNTs-absorbed form of pyrene. The indirect emission band is red-shifted with respect to the monomer band. Red-shifts of the position of the maximum from the monomeric form are ascribed to the interaction of the pyrene's $\pi$-electrons with other systems on which they are adsorbed onto [53]. The intermediate band position between the monomer and the excimer confirms that the pyrene contributing to the energy transfer process is the one adsorbed onto the nanotubes sidewalls.

\section{Conclusions}

In this work we have demonstrated that the realm of excitation energy-transfer can be extended to molecules whose emission spectrum does not significantly overlap with the absorption of SWNTs. This can be made possible by locally changing the behaviour of the electromagnetic fields in the immediate surrounding of the donor-acceptor pair. Plasmonic nanoparticles tethered to pyrene accomplish this task, enabling the transfer of energy from pyrene to the SWNTs, despite their non-overlapping emission-excitation spectra. In this way, we activated an excitation channel for all the nanotubes species present in a batch to emit. This channel works in the high-energy part of the solar spectrum, which cannot be covered by standard molecule-SWNT binary systems. Together with the near-field enhanced photoemission capability $[54,55,56]$, these results encourage the developement of further hybrid plasmonic nanosystems. Possible concepts could be exploiting plasmonic modes with higher symmetries to enhance the transfer rate between donor and acceptor which are not optimally aligned, or plasmonic particles with plasmons of different energies to activate an ensamble of donors of different nature on the same tube. This could be beneficial not only for solar energy collection but also for NIR-emission related applications as bio-imaging or night vision.

\section{Acknowledgments}

We gratefully thank the FU Focus Area NanoScale for financial support. Part of this work also has been granted by the SFB 658, subproject A6. We further thank S. Reich for her valuable support.

\section{References}

[1] A. Javey, J. Guo, Q. Wang, M. Lundstrom, and H. Dai. Ballistic carbon nanotube field-effect transistors. Nature 2003, 424, 654 . 
[2] F. Wang, G. Dukovic, LE. Brus, and TF. Heinz. The optical resonances in carbon nanotubes arise from excitons. Science 2005, 308, 838.

[3] J. Maultzsch, R. Pomraenke, S. Reich, E. Chang, D. Prezzi, A. Ruini, E. Molinari, M. S. Strano, C. Thomsen, and C. Lienau. Exciton binding energies in carbon nanotubes from two-photon photoluminescence. Phys. Rev. B 2005, 72, 241402.

[4] R. Saito, G. Dresselhaus, and MS. Dresselhaus. Physical Properties of Carbon Nanotubes. World Scientific 1998.

[5] S. Reich, C. Thomsen, and J. Maultzsch. Carbon Nanotubes: Basic Concepts and Physical Properties. Wiley-VCH 2007.

[6] X. Ma, N. F. Hartmann, S. K. Baldwin, J. K. S. amd Doorn, and H. Htoon. Room-temperature single-photon generation from solitary dopants of carbon nanotubes. Nature Nanotechnology 2015, 10, 671.

[7] P. Avouris, M. Freitag, and V. Perebeinos. Carbon-nanotube photonics and optoelectronics. Nature Photonics 2008, 2, 341.

[8] T. Gatti, S. Casaluci, M. Prato, M. Salerno, F. Di Stasio, A. Ansaldo, E. Menna, A. Di Carlo, and F. Bonaccorso. Boosting perovskite solar cells performance and stability through doping a poly3(hexylthiophene) hole transporting material with organic functionalized carbon nanostructures. Advanced Functional Materials 2016, 26, 7443.

[9] A. Graf, C. Murawski, Y. Zakharko, J. Zaumseil, and M.C. Gather. Infrared organic lightemitting diodes with carbon nanotube emitters. Advanced Materials 2017, 30, 1706711.

[10] M.J. O'Connell, S.M. Bachilo, C.B. Huffman, V.C. Moore, M.S. Strano, E.H. Haroz, K.L. Rialon, P.J. Boul, W.H. Noon, C. Kittrell, J. Ma, R.H. Hauge, R.B. Weisman, and R.E. Smalley. Band gap fluorescence from individual single-walled carbon nanotubes. Science 2002, 297, 593.

[11] F. Ernst, T. Heek, A. Setaro, R. Haag, and S. Reich. Energy transfer in nanotube-perylene complexes. Advanced Functional Materials 2012, 22, 3921.

[12] C. Roquelet, J.S. Lauret, V. Alain-Rizzo, C. Voisin, R. Fleurier, M. Delarue, D. Garrot, A. Loiseau, P. Roussignol, J.A. Delaire, and E. Deleporte. $\pi$-stacking functionalization of carbon nanotubes through micelle swelling. ChemPhysChem 2010, 11, $166 \%$.

[13] C. Roquelet, D. Garrot, J.S. Lauret, C. Voisin, V. Alain-Rizzo, Ph. Roussignol, J.A. Delaire, and E. Deleporte. Quantum efficiency of energy transfer in noncovalent carbon nanotube/porphyrin compounds. Applied Physics Letters 2010, 97, 141918.

[14] F. Ernst, T. Heek, A. Setaro, R. Haag, and S. Reich. Excitation characteristics of different energy transfer in nanotube-perylene complexes. Applied Physics Letters 2013, 102, 233105.

[15] F. Ernst, T. Heek, A. Setaro, R. Haag, and S. Reich. Functional surfactants for carbon nanotubes: Effects of design. The Journal of Physical Chemistry C 2013, 117, $115 \%$.

[16] F. Ernst, T. Heek, R. Haag, S. Reich, and A. Setaro. Chirally enhanced solubilization through perylene-based surfactant. physica status solidi (b) 2012, 249, 2465.

[17] C. Roquelet, F. Vialla, C. Diederichs, P. Roussignol, C. Delalande, E. Deleporte, J.S. Lauret, and C. Voisin. Local field effects in the energy transfer between a chromophore and a carbon nanotube: A single-nanocompound investigation. ACS Nano 2012, 6, 8796.

[18] G. Clave, G. Delport, C. Roquelet, J.S. Lauret, E. Deleporte, F. Vialla, B. Langlois, R. Parret, C. Voisin, P. Roussignol, B. Jousselme, A. Gloter, O. Stephan, A. Filoramo, V. Derycke, and S. Campidelli. Functionalization of carbon nanotubes through polymerization in micelles: A bridge between the covalent and noncovalent methods. Chemistry of Materials 2013, 25, 2700 . 
[19] F. Vialla, G. Delport, Y. Chassagneux, Ph. Roussignol, J.S. Lauret, and C. Voisin. Diameterselective non-covalent functionalization of carbon nanotubes with porphyrin monomers. Nanoscale 2016, 8, 2326.

[20] G. Delport, L. Orcin-Chaix, S. Campidelli, C. Voisin, and J.S. Lauret. Controlling the kinetics of the non-covalent functionalization of carbon nanotubes using sub-cmc dilutions in a co-surfactant environment. Nanoscale 2017, 9, 2646.

[21] A. Setaro. Advanced carbon nanotubes functionalization. Journal of Physics: Condensed Matter 2017, 29, 423003.

[22] R.J. Chen, Y. Zhang, D. Wang, and H. Dai. Noncovalent sidewall functionalization of singlewalled carbon nanotubes for protein immobilization. Journal of the American Chemical Society 2001, 123, 3838 .

[23] K. Yang, L.Z. Zhu, and B.S. Xing. Adsorption of polycyclic aromatic hydrocarbons by carbon nanomaterials. Environmental Science $\mathscr{E}$ Technology 2006, 40, textit1855.

[24] P. Bluemmel, A. Setaro, CS. Popeney, R. Haag, and S. Reich. Dispersion of carbon nanotubes using an azobenzene derivative. physica status solidi (b) 2010, 247, 2891.

[25] A. Setaro, P. Bluemmel, C. Maity, S. Hecht, and S. Reich. Non-covalent functionalization of individual nanotubes with spiropyran-based molecular switches. Advanced Functional Materials 2012, 22, 2425.

[26] V. Sgobba, G.M.A. Rahman, D.M. Guldi, N. Jux, S. Campidelli, and M. Prato. Supramolecular assemblies of different carbon nanotubes for photoconversion processes. Advanced Materials 2006, 18, 2264.

[27] F. D'Souza, A.S.D. Sandanayaka, and O. Ito. Swnt-based supramolecular nanoarchitectures with photosensitizing donor and acceptor molecules. The Journal of Physical Chemistry Letters 2010, 1, 2586.

[28] J. Bartelmess, B. Ballesteros, G. de la Torre, D. Kiessling, S. Campidelli, M. Prato, T. Torres, and D.M. Guldi. Phthalocyanine-pyrene conjugates: A powerful approach toward carbon nanotube solar cells. Journal of the American Chemical Society 2010, 132, 16202.

[29] C. Sartorio, V. Figà, P. Salice, D. Gragnato, S. Cataldo, M. Scopelliti, R. Improta, E. Menna, and B. Pignataro. Thiophene pyrenyl derivatives for the supramolecular processability of singlewalled carbon nanotubes in thin film heterojunction. Synthetic Metals 2017, 229, 7.

[30] T. Förster. Zwischenmolekulare Energiewanderung und Fluoreszenz. Annalen der Physik 1948, 2,55 .

[31] J. Li, SK. Cushing, F. Meng, T.R. Senty, A.D. Bristow, and N. Wu. Plasmon-induced resonance energy transfer for solar energy conversion. Nature Photonics 2015, 9, 601.

[32] L.Y. Hsu, W. Ding, and G.C. Schatz. Plasmon-coupled resonance energy transfer. The Journal of Physical Chemistry Letters 2017, 8, $235 \%$.

[33] L. Gabrielli, G. Altoe, M. Glaeske, S. Juergensen, S. Reich, A. Setaro, E. Menna, F. Mancin, and T. Gatti. Controlling the decoration of the reduced graphene oxide surface with pyrenefunctionalized gold nanoparticles. physica status solidi (b) 2017, 254, 1700281.

[34] P. Salice, A. Gambarin, N. Daldosso, F. Mancin, and E. Menna. Noncovalent interaction between single-walled carbon nanotubes and pyrene-functionalized gold nanoparticles in water-soluble nanohybrids. The Journal of Physical Chemistry C 2014, 118, 27028. 
[35] A. Setaro, C.S. Popeney, B. Trappmann, V. Datsyuk, R. Haag, and S. Reich. Polyglycerolderived amphiphiles for single walled carbon nanotube suspension. Chemical Physics Letters 2010, 493, $14 \%$.

[36] C.S. Popeney, A. Setaro, RC. Mutihac, P. Bluemmel, B. Trappmann, J. Vonneman, S. Reich, and R. Haag. Polyglycerol-derived amphiphiles for the solubilization of single-walled carbon nanotubes in water: A structure-property study. ChemPhysChem 2012, 13, 203.

[37] M.C. Daniel and D. Astruc. Gold nanoparticles: assembly, supramolecular chemistry, quantumsize-related properties, and applications toward biology, catalysis, and nanotechnology. Chemical Reviews 2004, 104, 293.

[38] R.H. Dicke. Coherence in spontaneous radiation processes. Phys. Rev. 1954, 93, 99.

[39] T. Brandes. Coherent and collective quantum optical effects in mesoscopic systems. Physics Reports 2005, 408, 315.

[40] V.N. Pustovit and T.V. Shahbazyan. Cooperative emission of light by an ensemble of dipoles near a metal nanoparticle: The plasmonic dicke effect. Phys. Rev. Lett. 2009, 102, 077401.

[41] V.N. Pustovit and T.V. Shahbazyan. Plasmon-mediated superradiance near metal nanostructures. Phys. Rev. B 2010, 82, 075429.

[42] H. Xu, J. Liu, X. Duan, J. Li, J. Xue, X. Sun, Y. Cai, ZK. Zhou, and X. Wang. Enhance energy transfer between quantum dots by the surface plasmon of ag island film. Opt. Mater. Express 2014, 4, 2586.

[43] A.A. Lyamkina, D.V. Dmitriev, A.I. Toropov, and S.P. Moshchenko. Non-radiative energy transfer in quantum dot ensemble mediated by localized surface plasmon. Applied Physics Letters 2010, 110, 011103.

[44] P.G. Wu and L. Brand. Resonance energy transfer: Methods and applications. Analytical Biochemistry 1994, 218, 1.

[45] M.W. Chu, V. Myroshnychenko, C.H. Chen, J.P. Deng, C.Y. Mou, and F.J. Garca de Abajo. Probing bright and dark surface-plasmon modes in individual and coupled noble metal nanoparticles using an electron beam. Nano Letters 2009, 9, 399.

[46] H. Qian, M. Zhu, Z. Wu, and R. Jin. Quantum sized gold nanoclusters with atomic precision. Accounts of Chemical Research 2012, 45, 1470.

[47] M. Zhou, C. Zeng, Y. Chen, S. Zhao, M.Y. Sfeir, M. Zhu, and R. Jin. Evolution from the plasmon to exciton state in ligand-protected atomically precise gold nanoparticles. Nature Communications 2016, 7, 13240.

[48] P. Bluemmel, A. Setaro, C. Maity, S. Hecht, and S. Reich. Tuning the interaction between carbon nanotubes and dipole switches: the influence of the change of the nanotube-spiropyran distance. Journal of Physics: Condensed Matter 2012, 24, 394005.

[49] E. Malic, A. Setaro, P. Bluemmel, C.F. Sanz-Navarro, P. Ordejon, S. Reich, and A. Knorr. Carbon nanotubes as substrates for molecular spiropyran-based switches. Journal of Physics: Condensed Matter 2012, 24, 394006.

[50] W.E. Acree Jr., S.A. Tucker, and J.C. Fetzer. Fluorescence emission properties of polycyclic aromatic compounds in review. Polycyclic Aromatic Compounds 1991, 2, 75.

[51] G.K. Bains, S.H. Kim, E.J. Sorin, and V. Narayanaswami. The extent of pyrene excimer fluorescence emission is a reflector of distance and flexibility: Analysis of the segment linking the ldl receptor-binding and tetramerization domains of apolipoprotein e3. Biochemistry 2012, 51, $620 \%$. 
[52] PY. Yu and M. Cardona. Fundamentals of Semiconductors - Physics and Materials Properties. Springer-Verlag Berlin Heidelberg 2010.

[53] P. Koenig, S.A. Reines, and C.R. Cantor. Pyrene derivatives as fluorescent probes of conformation near the 3'termini of polyribonucleotides. Biopolymers 1977, 16, 2231.

[54] M. Glaeske and A. Setaro. Nanoplasmonic colloidal suspensions for the enhancement of the luminescent emission from single-walled carbon nanotubes. Nano Research 2013, 6, 593.

[55] J. Yang, Q. Zhao, M. Lyu, Z. Zhang, X. Wang, M. Wang, Z. Gao, and Y. Li. Chirality-selective photoluminescence enhancement of ssdna-wrapped single-walled carbon nanotubes modified with gold nanoparticles. Small 2015, 12, 3164.

[56] M. Glaeske, M. Kumar, T. Bisswanger, S. Vaitiekenas, C. Soci, R. Narula, A. Bruno, and A. Setaro. Relaxation lifetimes of plasmonically enhanced hybrid gold-carbon nanotubes systems. Nanotechnology 2017, 28, 255202. 